\title{
On Optimal Control Model for the Treatment of Dual HIV-Parasitoid Pathogen Infection
}

\section{Bassey $\mathrm{EB}^{1 *}$, Kimbir RA ${ }^{2}$ and Lebedev $\mathrm{KA}^{3}$}

${ }^{1}$ Department of Mathematical/Statistics, Cross River University of Technology, Calabar, Nigeria

${ }^{2}$ Department of Mathematics, Statistics and Computer Science, Federal University of Agriculture, Makurdi, Nigeria

${ }^{3}$ Department of Computational Mathematics and Informatics, Kuban State University, Krasnodar, Russia

\begin{abstract}
Following the insurmountable and seeming incurable status for the most acclaimed infectious disease - HIV, and which have been worsened by its allies of infectious diseases, this paper projected using ordinary differential equations, a 4-Dimensional mathematical model that accounted for the percentage optimal benefits and the methodological application of chemotherapy - RTI, in the interaction of dual HIV- parasitoid pathogen infectivity with the human immune system. Simple analytical optimal control method was deployed, primed by the maximization of healthy immune system on the basis of control effect of chemotherapy on viruses' infectivity. Using Pontryagin's Maximum Principle, the study established the model dynamical optimal control as a composition of system state variables, coupled with four adjoint systems with corresponding initial and transversality conditions together with the optimal control function. The model was solved numerically and results indicated thus: benefits on cost function as highest when onset of infection were followed by high intensity chemotherapy schedule; while optimum control were achieved with prolong treatment administration. The study revealed that optimal control is a function of dynamic optimal weight factor and is independent of prolong treatment duration. The study therefore, advocates the incorporation of dual immunotherapies for the treatment of multiple virus infectivity.
\end{abstract}

Keywords: Adjoint-system; Chemotherapy; Immunotherapies; Optimal-benefits; Transversality-conditions; Methodological; Virusesinfectivity

\section{Introduction}

Until a clear and decisive medical procedure is formulated for the most dominated infectious disease - human immune deficiency virus (HIV), which often transmute into acquired immune deficiency syndrome (AIDS) and its affiliated diseases, the search into its suppressive and preventive remedies, remain a task for this generation of scientific researchers. In reality, it is evident to appreciate recent multiplicities of new cases of HIV epidemic and its associated infectivity $[1,2]$. Common among the dual infectivity includes: HIV-parasitoid pathogen, HIV-tuberculosis, HIV- hepatitis C, etc. [3,4].

Currently, in the absent of cure, suppression and prevention has been the major anchor of control. Effective management of dual HIVpathogenic infected patients requires progressive administration of prescribed chemotherapy, a process that involves clinical modeling [5]. Appreciating the role of chemotherapies, the models [6,7], studied the control of effect of HI-virus infectivity on immune system using AZT, which acts as reverse transcription inhibitor resulting to the interruption of key stages of infection process. Using reverse transcriptase inhibitor (RTI) as single treatment, [8] studied the optimal control strategy for a fully determined HIV model aimed at clinical testing and monitoring of HIV/AIDS diseases; as Optimal Control of HIV Infection by using Fuzzy Dynamical Systems had been investigated by [9]. The model demonstrated the $\mathrm{CD} 4^{+} \mathrm{T}$ cells measurement and viral load count. The investigation on the analysis based on the quasi-steady state of the asymptomatic period before it is disturbed by chemotherapy can be found in [10]. The application of highly anti-retroviral therapy (HAART) regimen in the treatment and suppression of viral replication and immune system recovery was conducted by $[5,11]$.

In this paper, we propose using ordinary differential equation (ODE), the formulation of 4-Dimensional mathematical model that accounts for the optimal benefits and methodological treatment of dual HIV-parasitoid pathogen infectivity on the host $-\mathrm{CD} 4^{+} \mathrm{T}$ cells, with reverse transcriptase inhibitor (RTI) as a treatment factor. The investigation is presented as an optimal problem with the assumption that, the regulation of the chemotherapy directly controls the infectivity of these dual viruses against the immune system. Unlike AZT, the clinical choice for RTI is based on the dual exclusive suppressing and eliminating capability of the inhibitor on dual infectious viruses $[5,12]$. The present study propose the use of Pontryagin's Maximum Principle in the analysis of the optimal control state and results numerically illustrated via Runge-Kutter of order of precision 4 in a Mathcad environment.

The propositions and applications of optimal control strategies in the study of interaction of chemotherapies and viral load within the immune system had been presented in 2-Dimensional model by $[13,14]$; in 3 -Dimensional model by $[6,12]$ and in 5 -Dimensional model by [11], where here, it was established to be incompatible using discretization technique, following large error derivatives. Against the above structures, this present model is ultimately vested in the study of a 4-Dimensional differential equation with problem statement argued along optimal control strategy.

The explicit dimension of this model is the investigation of the

*Corresponding author: Bassey EB, Department of Mathematical/Statistics, Cross River University of Technology, Calabar 540252, Nigeria, Tel: +234(806)7535050; E-mail: awaserex@ymail.com

Received: December 09, 2016; Accepted: December 13, 2016; Published: December 25, 2016

Citation: Bassey EB, Kimbir RA, Lebedev KA (2016) On Optimal Control Mode for the Treatment of Dual HIV-Parasitoid Pathogen Infection. J Bioengineer \& Biomedical Sci 7: 212. doi: 10.4172/2155-9538.1000212

Copyright: (c) 2016 Bassey EB, et al. This is an open-access article distributed under the terms of the Creative Commons Attribution License, which permits unrestricted use, distribution, and reproduction in any medium, provided the original author and source are credited. 
periodic treatment schedules, taking into account, the definitive time limit before the resistivity habit of chemotherapy. The time limits of most chemotherapy have been identified in $[1,11,13-16]$. Thus, we intend to front an optimal control statutory model which demonstrates the connective interplay of chemotherapy (RTI) on HIV-pathogen and the blood plasma with the aim of maximizing the objective functional as a basis for accessing $\mathrm{CD} 4^{+} \mathrm{T}$ cells progression and the simultaneous outcome of probable reduction in systemic cost [7].

Devotedly, this paper is organized as follows: section 1, which been covered by the introductory aspect of the work, we introduce in section 2, the material and methods, which consists of the formulation of the model as a problem statement, the proposed design optimization control strategy and the Pontryagin's Maximum Principle as analysis method. In section 3, we present a number of numerical computations to illustrate the efficiency and reliability of the method as well as the discussions. Finally, the paper's conclusion and remarks are carefully drawn in section 4 .

\section{Material and Methods}

We constitute this section with the problem statement and model formulation; and the designed optimal control strategy analyze as a function of the Pontryagin's Maximum Principle.

\section{Problem statement and model formulation}

Here, we assume the dual viruses infect the same $\mathrm{CD} 4^{+} \mathrm{T}$ cells therefore, using figure 1 below, we develop via ordinary differential equation, a 4-Dimensional mathematical model defined as problem statement of optimal control problem, targeted to account for the optimal methodology of treatment of dual HIV-parasitoid pathogen infections.

From Figure 1 above, if the concentration of the various subgroups under consideration represent the population number per unit volume, $m m_{3}$, then $U_{T}$ - uninfected $\mathrm{CD} 4^{+} \mathrm{T}$ cell count, $I_{T}$ - infected $\mathrm{CD} 4^{+}$ T cells (by both viruses), $V$ - free viral load and $P$ - parasitoid pathogen, represent the biological interaction of the variables.

The physiological definition of the model is the dynamic derivation of the following differential equations:

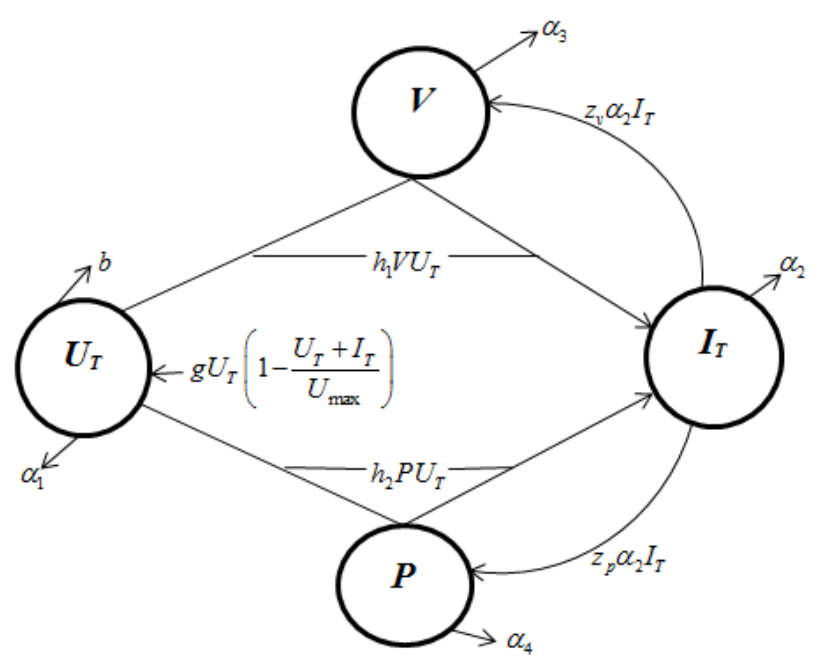

Figure 1: Schematic diagram for dual HIV-pathogen infections.

$$
\begin{aligned}
\frac{d U_{T}}{d t} & =\frac{b}{1+V+P}+g U_{T}\left(1-\frac{U_{T}+I_{T}}{U_{\max }}\right)-\alpha_{1} U_{T}-h_{1} V U_{T}-h_{2} P U_{T} \\
\frac{d I_{T}}{d t} & =h_{1} V U_{T}+h_{2} P U_{T}-\left(z_{v}+z_{p}\right) \alpha_{2} I_{T} \\
\frac{d V}{d t} & =z_{v} \alpha_{2} I_{T}-\alpha_{3} V U_{T} \\
\frac{d P}{d t} & =z_{p} \alpha_{2} I_{T}-\alpha_{4} P U_{T}
\end{aligned}
$$

With initial conditions:

$$
U_{T}(0)=U_{(T) 0}, I_{T}(0)=I_{(T) 0}, V(0)=V_{0}, P(0)=P_{0}
$$

and satisfying the biological variables and parameters values as define in (Table 1).

Explicitly, the epidemiological interpretation of the model equations (2.1)-(2.4) can be deduce as follows: In equation (2.1), the function $b / 1+V+P$, is the source term of uninfected $\mathrm{CD} 4^{+} \mathrm{T}$ cells, differentiated with respect to the invasion by the external viruses; $g$, is the $\mathrm{CD} 4^{+} \mathrm{T}$ cell growth rate (per day), having a logistic term $\left(1-U_{T}+I_{T} /\right.$ $\left.U_{\max }\right)$. This shows that $U_{T}$ is always within the range of $U_{\max }$. Upon exposure to $V$ and $P$, the $C D 4^{+} \mathrm{T}$ cells $\left(U_{T}\right)$, becomes infected and loss with magnitude of $h_{1} V U_{T}$ and $h_{2} P U_{T}$ respectively. Moreso $U_{T}$, is life cycle bound by natural death rate of $a_{1}$. From equation (2.2), the term $h_{1} V U_{T}$ and $h_{2} P U_{T}$ model the rate at which free viral load and parasitoidpathogen infects the $\mathrm{CD} 4^{+} \mathrm{T}$ cells and having $a_{1} I_{T}$, death rate with $z_{v}$ and $z_{p}$, rate of replications of the viruses before infected $\mathrm{CD} 4^{+} \mathrm{T}$ cells host dies. Taking equation (2.3), the term $z_{v} a_{1} I_{T}$, represent the rate at which viral load is produced by infected $\mathrm{CD} 4^{+} \mathrm{T}$ cells into the viral load compartment. The indicator $a_{3}$, is the loss of viral load infected $\mathrm{CD} 4^{+} \mathrm{T}$ cells. Finally, in equation (2.4), $z_{p_{2}} I_{T}$ is the rate of production of parasitoid-pathogen by infected $\mathrm{CD} 4^{+} \mathrm{T}$ cells, and $a_{4}$, is the loss of pathogen infected $\mathrm{CD} 4^{+} \mathrm{T}$ cells. Other closely related HIV - infection models can be readily view from $[1,6,14]$.

Furthermore, the application of chemotherapy and its effects on the model can be adduced by multiplying the terms $h_{1} V U_{T}$ and $h_{2} P U_{T}$ from equations (2.1)-(2.4), by the function $r(t)$, which initiate our optimal control design.

\section{Optimization control strategy for chemotherapy}

Since prime interest is on the maximization of healthy $\mathrm{CD}^{+} \mathrm{T}$

\begin{tabular}{|l|c|}
\hline Dependent Variables & Initial values \\
\hline $\mathrm{U}_{\mathrm{T}}$ Uninfected CD4+ T Cell population & $0.6 \mathrm{~mm}^{-3}$ \\
\hline $\mathrm{I}_{\mathrm{T}}$ Infected CD4+ T Cell population & 0.0 \\
\hline $\mathrm{V}$ Infectious HIV (Viral load) population & $0.2 \mathrm{~mm}^{-3}$ \\
\hline $\mathrm{P}$ Infectious parasitoid-pathogen population & $0.1 \mathrm{~mm}^{-3}$ \\
\hline Parameters and Constants & Values \\
\hline $\mathrm{b}$ Natural Source of uninfected CD4+ T Cell & $0.02 \mathrm{~mm}^{-3} \mathrm{~d}^{-1}$ \\
\hline$a 1$ Natural death rate of uninfected CD4+ T Cell & $0.2 \mathrm{~d}^{-1}$ \\
\hline $\mathrm{a}_{2}$ death rate of infected CD4+ T Cell & $0.5 \mathrm{~d}^{-1}$ \\
\hline $\mathrm{a}_{3}$ death rate free viral load, V & $0.4 \mathrm{~d}^{-1}$ \\
\hline $\mathrm{a}_{4}$ death rate free parasitoid pathogens, $P$ & $0.5 \mathrm{~d}^{-1}$ \\
\hline $\mathrm{h}_{1}$ rate CD4+ T cells becoming infected by free virus, V & $0.044 \mathrm{~mm}^{-3} \mathrm{~d}^{-1}$ \\
\hline $\mathrm{h}_{2}$ rate CD4+ T cells becoming infected pathogens, $\mathrm{P}$ & $0.016 \mathrm{~mm}^{-3} \mathrm{~d}^{-1}$ \\
\hline $\mathrm{g}$ rate of growth of CD4+ T cell population & $0.04 \mathrm{~d}^{-1}$ \\
\hline$z \gamma$ Number of replication of HI-Virus by IT cells & 0.5 \\
\hline$z \gamma$ Number of replication of P-pathogen by IT cells & 0.3 \\
\hline Umax maximum level of CD4+ T cells population & $0.8 \mathrm{~mm}^{-3}$ \\
\hline
\end{tabular}

Table 1: Variables and parameters for optimal control treatment $0.044 \mathrm{U}$. 
cells from the control effect of chemotherapy application, then we investigate the percentage effect of the chemotherapy on the biological interactions of the $\mathrm{CD} 4^{+} \mathrm{T}$ cells and the dual infectivity of the viruses $(V$ and $P$ ). We denote this control function by $r(t)$ with, which we multiply, the parameters $h_{1}$ and $h_{2}$ of equations (2.1) and (2.2); the proceedings, which are guided by the following assumption:

Assumption 1: The model control class designated by $r(t)$, is a measurable function defined on the interval $t \in\left[t_{0}, t_{f}\right]$ and having the domain $0 \leq r(t) \leq 1$.

This assumption defines the treatment interval following the allowable window of treatment by chemotherapy and the anticipated cogent result before mutations and development of drug resistance by HI-virus and pathogen [17]. Moreover, drug side-effects as a function of treatment duration are arguably accounted for. Therefore, we consider $t \in\left[t_{0}, t_{f}\right] \leq 30$ months [11], and define the state system as:

$$
\begin{aligned}
& \frac{d U_{T}}{d t}=\frac{b}{1+V+P}+g U_{T}\left(1-\frac{U_{T}+I_{T}}{U_{\max }}\right)-\alpha_{1} U_{T}-r(t)\left[h_{1} V U_{T}+h_{2} P U_{T}\right] \\
& \frac{d I_{T}}{d t}=r(t)\left[h_{1} V U_{T}+h_{2} P U_{T}\right]-\left(z_{v}+z_{p}\right) \alpha_{2} I_{T} \\
& \frac{d V}{d t}=z_{v} \alpha_{2} I_{T}-\alpha_{3} V U_{T} \\
& \frac{d P}{d t}=z_{p} \alpha_{2} I_{T}-\alpha_{4} P U_{T}
\end{aligned}
$$

and having initial values for $U_{T}, I_{T}, V, P$ at $t_{0}$.

The objective functional that maximizes the control system is defined as:

$$
Q(\mathrm{r})=\int_{t_{0}}^{t_{f}}\left[U_{T}(t)-\frac{1}{2} \psi(1-r(t))^{2}\right] d t
$$

Where, $\psi$ is the optimal weight factor, which maximizes the benefit based on the $\mathrm{CD} 4^{+} \mathrm{T}$ cells and minimizes the systemic cost of chemotherapy based on the percentage effect given by $(1-r(t))$. It's obvious that if $r(t)=0$, as the maxima drug usage, then the maximal cost is given by $(1-r(t))^{2}$. The introduction of the parameter $\psi \geq 0$, designated as optimal weight factor, follows from the fact that, the benefit to the cost functional is nonlinear. Hence, the need to introduce a simple non-linear control on the cost indicator becomes obvious. We therefore characterize the optimal control $r^{*}$, of the objective functional to satisfy the expression:

$$
\max _{0 \leq r \leq 1} Q(r)=Q(r *)
$$

Thus, $Q(r *)=\left\{Q(r)|r \in A ; A=r| r\right.$, measurable, $\left.\forall t \in\left[t_{0}, t_{f}\right]\right\}, \quad$ is the measurable control set. The penalty term for the constraints of the objective functional is the Hamiltonian arguments define by the Lagrangian. That is,

$$
\begin{aligned}
& L\left(U_{T}(t), I_{T}(t), V(t), P(t), r(t), \lambda_{1}(t), \lambda_{2}(t), \lambda_{3}(t), \lambda_{4}(t)\right) \\
& =U_{T}(t)-\frac{1}{2} \psi(1-r(t))^{2} \\
& +\lambda_{1}\left[\frac{b}{1+V+P}+g U_{T}\left(1-\frac{U_{T}+I_{T}}{U_{\max }}\right)-\alpha_{1} U_{T}-r(t)\left(h_{1} V U_{T}+h_{2} P U_{T}\right)\right] \\
& +\lambda_{2}\left[r(t)\left(h_{1} V U_{T}+h_{2} P U_{T}\right)-\left(z_{v}+z_{p}\right) \alpha_{2} I_{T}\right] \\
& +\lambda_{3}\left[z_{v} \alpha_{2} I_{T}-\alpha_{3} V U_{T}\right]+\lambda_{4}\left[z_{p} \alpha_{2} I_{T}-\alpha_{4} P U_{T}\right] \\
& +k_{1}(t) r(t)+k_{2}(t)(1-r(t))
\end{aligned}
$$

Where,

$k_{1}(t) \geq 0, \quad k_{2}(t) \geq 0$, are the penalty multipliers satisfying $k_{1}(t) \mathrm{r}(\mathrm{t})=0$ and $k_{2}(t)(1-r(t))=0$. Thus, the maximum principle $[1,6,18]$, gives the existence of adjoint variables satisfying:

$$
\begin{aligned}
& \frac{d \lambda_{1}}{d t}=-\frac{\partial L}{\partial U_{T}}=-\left[\begin{array}{l}
1+\lambda_{1}\left(-\alpha_{1}+g\left(1-\frac{\left(2 U_{T}+I_{T}\right)}{U_{\max }}\right)-r(t)\left(h_{1} V+h_{2} P\right)\right. \\
+\lambda_{2} r(t)\left(h_{1} V+h_{2} P\right)-\lambda_{3} \alpha_{3} V-\lambda_{4} \alpha_{4} P
\end{array}\right] \\
& \frac{d \lambda_{2}}{d t}=-\frac{\partial L}{\partial I_{T}}=-\left[-\frac{\lambda_{1} g U_{T}}{U_{\max }}-\lambda_{2}\left(z_{v}+z_{p}\right) \alpha_{2}+\lambda_{3} z_{v} \alpha_{2}+\lambda_{4} z_{p} \alpha_{2}\right] \\
& \frac{d \lambda_{3}}{d t}=-\frac{\partial L}{\partial V}=-\left[\lambda_{1}\left(-\frac{b}{(1+v)^{2}}-r(t)\left(h_{1} U_{T}\right)\right)+\lambda_{2} r(t) h_{1} U_{T}-\lambda_{3} \alpha_{3} U_{T}\right] \\
& \frac{d \lambda_{4}}{d t}=-\frac{\partial L}{\partial P}=-\left[\lambda_{1}\left(-\frac{b}{(1+P)^{2}}-r(t)\left(h_{2} U_{T}\right)\right)+\lambda_{2} r(t) h_{2} U_{T}-\lambda_{4} \alpha_{4} U_{T}\right]
\end{aligned}
$$

Where,

$\lambda_{i}\left(t_{f}\right)=0$ for $i=1, . ., 4$, are the transversality conditions.

Now, since

$$
\begin{aligned}
& L=\left(-\frac{1}{2} \psi(1-r(\mathrm{t}))^{2}\right)-\lambda_{1}\left(h_{1} V U_{T}+h_{2} P U_{T}\right)+\lambda_{2} r(t)\left(h_{1} V U_{T}+h_{2} P U_{T}\right) \\
& +k_{1}(t) r(t)+k_{2}(t)(1-r(t))+\text { terms, } \ldots \text { without } \ldots r,
\end{aligned}
$$

differentiating this expression for $L$ with respect to $r$, gives:

$$
\frac{\partial L}{\partial r}=\left(h_{1} V U_{T}+h_{2} P U_{T}\right)\left(\lambda_{2}-\lambda_{1}\right)+\psi(1-\mathrm{r})+\mathrm{k}_{1}(\mathrm{t})-\mathrm{k}_{2}(\mathrm{t})=0 .
$$

Solving for optimal control, we have

$$
r^{*}(t)=\frac{\left(\lambda_{2}-\lambda_{1}\right)\left(h_{1} V U_{T}+h_{2} P U_{T}\right)+k_{1}(t)-\mathrm{k}_{2}(\mathrm{t})+\psi}{\psi}
$$

Then, we can examine the expression for $r^{*}$ by taking into consideration the following 3 cases:

On the set $\left\{t \mid 0<r^{*}(t)<1\right\}: k_{1}(t)=k_{2}(t)=0$, and we obtain the optimal control as:

$$
r^{*}(t)=\frac{\left(\lambda_{2}-\lambda_{1}\right)\left(h_{1} V U_{T}+h_{2} P U\right)+\psi}{\psi} .
$$

On the set $\left\{t \mid r^{*}(t)=1\right\}: k_{1}(t)=0, k_{2}(t) \geq 0$, hence

$$
\begin{aligned}
& r^{*}(t)=1=\frac{\left(\lambda_{2}-\lambda_{1}\right)\left(h_{1} V U_{T}+h_{2} P U\right)-\mathrm{k}_{2}(\mathrm{t})}{\psi}+1, \text { which implies } \\
& 0 \leq k_{2}(t)=\left(\lambda_{2}-\lambda_{1}\right)\left(\mathrm{h}_{1} \mathrm{VU}_{T}+h_{2} P U_{T}\right)
\end{aligned}
$$

and

$$
1 \leq \frac{\left(\lambda_{2}-\lambda_{1}\right)\left(h_{1} V U_{T}+h_{2} P U\right)+\psi}{\psi} .
$$

On the set $\left\{t \mid r^{*}(t)=0\right\}: k_{2}(t)=0, k_{2}(t) \geq 0$. Hence, the optimal control is:

$$
r^{*}(t)=\frac{\left(\lambda_{2}-\lambda_{1}\right)\left(h_{1} V U_{T}+h_{2} P U\right)+k_{1}(t)+\psi}{\psi}=0 .
$$

Therefore, $k_{1}(t) \geq 0$ implies that $\frac{\left(\lambda_{2}-\lambda_{1}\right)\left(\mathrm{hVU}_{T}+h P U_{T}\right)+k_{1}(t)+\psi}{\psi} \leq 0$, which implies 


$$
r^{*}(t)=\left(\frac{\left(\lambda_{2}-\lambda_{1}\right)\left(h_{1} V U_{T}+h_{2} P U\right)+\psi}{\psi}\right)^{+}=0 .
$$

Thus, the optimal control is characterized by the combination of these 3 cases satisfying the equation:

$$
r^{*}=\min \left(\left(\frac{\left(\lambda_{2}-\lambda_{1}\right)\left(h_{1} V U_{T}+h_{2} P U\right)+\psi}{\psi}\right)^{+}, 1\right)
$$

Where,

$$
\begin{aligned}
& \left(\frac{\left(\lambda_{2}-\lambda_{1}\right)\left(h_{1} V U_{T}+h_{2} P U\right)+\psi}{\psi}\right)^{+} \\
& =\left\{\begin{array}{ccc}
\frac{\left(\lambda_{2}-\lambda_{1}\right)\left(h_{1} V U_{T}+h_{2} P U\right)}{\psi}+1 & \text { if } & \left(\lambda_{2}-\lambda_{1}\right)\left(\mathrm{h}_{1} \mathrm{VU}_{T}+h_{2} P U_{T}\right)+\psi>0 \\
0 & \text { if } & \left(\lambda_{2}-\lambda_{1}\right)\left(\mathrm{h}_{1} \mathrm{VU}_{T}+h_{2} P U_{T}\right)+\psi \leq 0 .
\end{array}\right.
\end{aligned}
$$

It follows that if $\left(\lambda_{2}-\lambda_{1}\right)<0$ for somet, then $r^{*}(t) \neq 1$ and we say $0 \leq r^{*}(t)<1$ for thoset, which imply treatment initiation. So, it becomes obvious that control depends on the adjoints $\lambda_{1}$ and $\lambda_{2}$, in view of that fact that the adjoints corresponds to the state variables $U_{T}$ and $I_{T}$, as in the first two state equations, which contains the control terms. Therefore, we see that the optimization control system is define by the state system (2.5)-(2.8), coupled with the adjoint system (2.11)(2.14) with corresponding initial and transversally conditions and by substituting in the expression (2.15) for $r^{*}$ in equations (2.5), (2.6), (2.11), (2.13) and (2.14). Thus, utilizing equation (2.15) for $r^{*}$ we obtain the dynamic optimal control as:

$$
\begin{aligned}
& \frac{d U_{T}}{d t}=\frac{b}{1+V+P}+g U_{T}\left(1-\frac{U_{T}+I_{T}}{U_{\max }}\right) \\
& -\alpha_{1} U_{T}-\min \left(\left(\frac{\left(\lambda_{2}-\lambda_{1}\right)\left(\mathrm{h}_{1} \mathrm{VU}_{T}+h_{2} P U_{T}\right)+\psi}{\psi}\right)^{+}, 1\right) \cdot\left[h_{1} V U_{T}+h_{2} P U_{T}\right] . \\
& \frac{d I_{T}}{d t}=\min \left(\left(\frac{\left(\lambda_{2}-\lambda_{1}\right)\left(\mathrm{h}_{1} \mathrm{VU}_{T}+h_{2} P U_{T}\right)+\psi}{\psi}\right)^{+}, 1\right) \cdot\left[h_{1} V U_{T}+h_{2} P U_{T}\right]-\left(z_{v}+z_{p}\right) \alpha_{2} I_{T} \\
& \frac{d V}{d t}=z_{v} \alpha_{2} I_{T}-\alpha_{3} V U_{T} \\
& \frac{d P}{d t}=z_{p} \alpha_{2} I_{T}-\alpha_{4} P U_{T}
\end{aligned}
$$

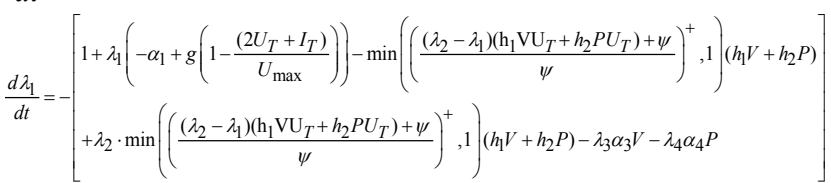

$$
\begin{aligned}
& \frac{d \lambda_{2}}{d t}=-\left[-\frac{\lambda_{1} g U_{T}}{U_{\max }}-\lambda_{2}\left(z_{v}+z_{p}\right) \alpha_{2}+\lambda_{3} z_{v} \alpha_{2}+\lambda_{4} z_{p} \alpha_{2}\right] \text {. } \\
& \frac{d \lambda_{3}}{d t}=-\left\{\begin{array}{l}
\lambda_{1}\left[-\frac{b}{(1+v)^{2}}-\min \left(\left(\frac{\left(\lambda_{2}-\lambda_{1}\right)\left(\mathrm{h}_{1} \mathrm{VU}_{T}+h_{2} P U_{T}\right)+\psi}{\psi}\right)^{+}, 1\right)\left(h_{1} U_{T}\right)\right. \\
+\lambda_{2}\left[\min \left(\left(\frac{\left(\lambda_{2}-\lambda_{1}\right)\left(\mathrm{h}_{1} \mathrm{VU}_{T}+h_{2} P U_{T}\right)+\psi}{\psi}\right)^{+}, 1\right) h_{1} U_{T}\right]-\lambda_{3} \alpha_{3} U_{T}
\end{array}\right\} \\
& \frac{d \lambda_{4}}{d t}=-\left\{\begin{array}{l}
\lambda_{1}\left[-\frac{b}{(1+P)^{2}}-\min \left(\left(\frac{\left(\lambda_{2}-\lambda_{1}\right)\left(\mathrm{h}_{1} \mathrm{VU}_{T}+h_{2} P U_{T}\right)+\psi}{\psi}\right)^{+}, 1\right)\left(h_{2} U_{T}\right)\right] \\
+\lambda_{2}\left[\min \left(\left(\frac{\left(\lambda_{2}-\lambda_{1}\right)\left(\mathrm{h}_{1} \mathrm{VU}_{T}+h_{2} P U_{T}\right)+\psi}{\psi}\right)^{+}, 1\right) h_{2} U_{T}\right]-\lambda_{4} \alpha_{4} U_{T}
\end{array}\right\}
\end{aligned}
$$

So that, $\lambda_{i}\left(t_{f}\right)=0$ for $i=1, . ., 4$ and $U_{T}(0)=U_{(T) 0}, I_{T}(0)=I_{(T) 0}, V(0)=V_{0}, P(0)=P_{0}$. It is of

interest that the existence and uniqueness of optimal control system is of standard result, which can be found in $[1,13]$.

\section{Numerical Simulations and Discussion}

Here, we put forward a number of numerical computations to illustrate the efficiency and reliability of the method as well as the results analyses. Using the parameter values as in Table 1 , and with the aid of Runge-Kutter of order of precision 4, in a Mathcad platform, we simulate for the initial values for the $\mathrm{T}$ cells, infected cell, viral load and the pathogen, without chemotherapy treatment. This task is accomplished using the basic model equations (2.1)-(2.4). The numerical results are then deployed to establish the different treatment initial conditions.

\section{Numerical simulations}

Figures $2 \mathrm{a}-2 \mathrm{~d}$ below represents initial simulation of the basic model equations (2.1)-(2.4), without treatment. We observed from figure $2 \mathrm{~b}$, that infection was sharp and highest at the $3^{\text {rd }}$ month (i.e., $\left.I_{T}(3)=5.92 \times 10^{-3}\right)$, which correspond to decrease in healthy $C D 4^{+}$ $\mathrm{T}$ cells at a value 0.38 , as in figure $2 \mathrm{a}$. Therefore, $U_{T}(3)=0.38$, is the minimum count of $\mathrm{CD}^{+} \mathrm{T}$ cells, with which treatment is initiated. The sharp decline of infected T cells after 3 months is an indication of the intensity of the combined infectivity of the viruses on the $\mathrm{T}$ cells. Due to fast replications of the viruses into the blood plasma, we notice de-transmutation of the viruses, such that $V(3)=0.12$ and $P(3)=0.051$. Figures $2 \mathrm{c}$ and $2 \mathrm{~d}$.correspond to decline of both viral load and parasitoid-pathogen following their transmission and infection of healthy $\mathrm{CD}^{+} \mathrm{T}$ cells but showing persistent resistivity throughout the duration of investigation, with viral load more acute.

Using the outcome of values of the variables from the initial simulation as in figures $2 \mathrm{a}-2 \mathrm{~d}$.

Above, the optimality system is solved following the application of chemotherapy in an observed period of 30 months of treatment. We simulate as in figures 3a-3d below, equations (2.5)-(2.8) representing the initiation of chemotherapy with $r(t)=0.5$, as the treatment control function. The benefit (objective) functional $Q(r)$, as in equation (2.9), corresponding to the application of the chemotherapy is simulated as in figure 4.

From Figure 3a below, with chemotherapy control function at $r(t)=0.5$, such that treatment benefit is describe by growth rate in $\mathrm{CD} 4^{+}$ $\mathrm{T}$ cells $(g=0.8)$, increase in viruses clearance rate $\left(a_{2}=0.52, a_{3}=0.45\right.$, $\left.a_{4}=0.54\right)$; and decline of $a_{1}=0.02$, as natural death rate of CD $4^{+} \mathrm{T}$ cells, we observe a maximization of the healthy $\mathrm{CD} 4^{+} \mathrm{T}$ cells, which increase tremendously after 27 months of drug application i.e., $U_{T}(27)=0.802$. Simulation also indicates drastic decline of infected $\mathrm{CD} 4^{+} \mathrm{T}$ cells i.e., $I_{T}(27)$ near zero, as inscribe by figure $3 \mathrm{~b}$. The de-mutations of viral load and pathogen following the application of chemotherapy, saw diminishment of HI-virus to near zero after 21 months and 19 months for parasitoid-pathogen (Figures $3 \mathrm{c}$ and $3 \mathrm{~d}$ ).

From Figure 4 below, using Equation (2.9), we simulate the objective functional $Q(r)$, corresponding to the applied chemotherapy treatment. Here, we aim at analyzing the magnitude of the systemic cost of treatment within drug validity. It is seen that initiation of treatment commenced with high intensity chemotherapy (i.e., $\psi=10$ and $r(t)=0.5$ ), which gradually approach stability after 27 months of prolong administration. 


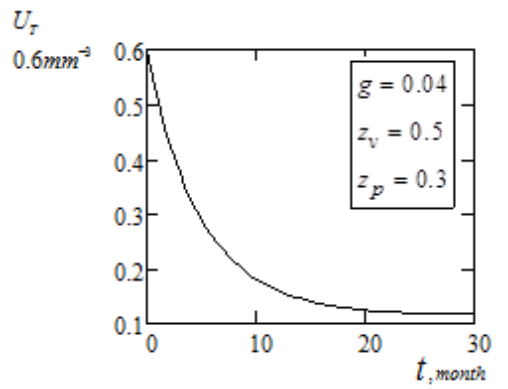

a) Simulation of uninfected $\mathrm{CD}^{+} \mathrm{T}$ cells, $U_{\tau}=0.6$ V

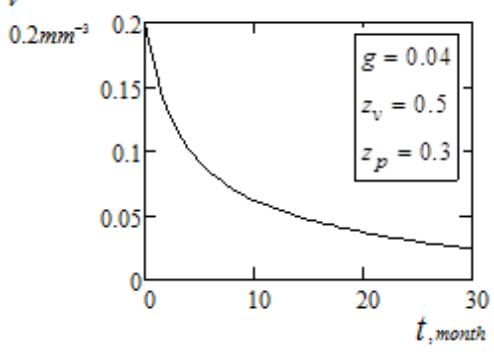

c) Simulation of viral load, $V=0.2$

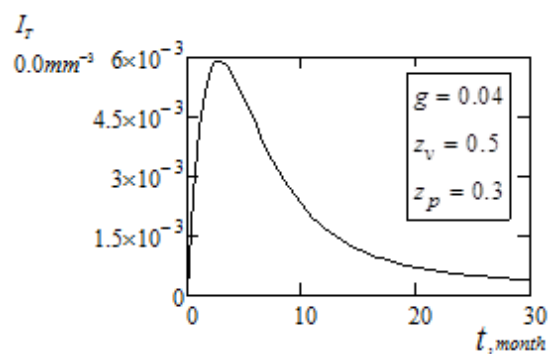

b) Simulation of viruses infected CD4 $\mathrm{T}$ cells, $I_{T}=0.0$

$P$

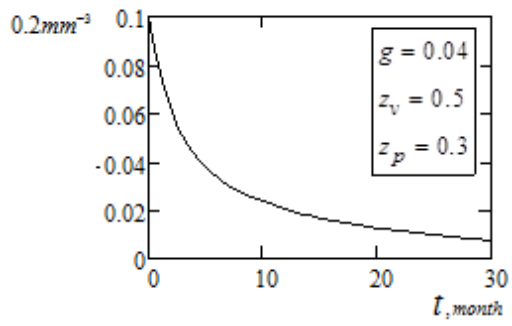

d) Simulation of parasitoid-pathogen, $P=0.2$

Figure 2: Simulation of dual HIV-pathogen infection without treatment.

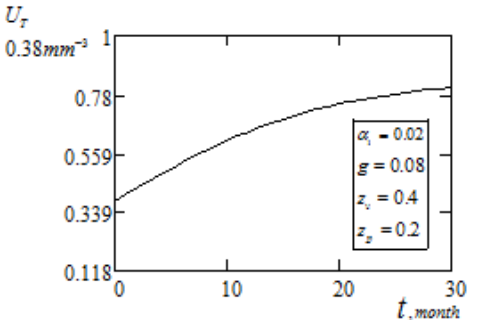

a) Uninfected $\mathrm{CD} 4+\mathrm{T}$ cells with treatment, $U_{T}(3)=0.38$

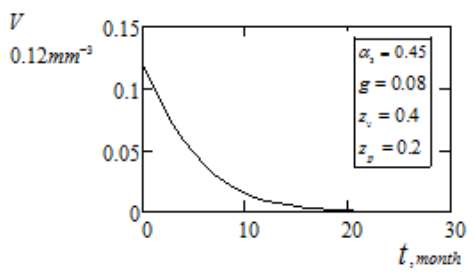

c) Viral load with treatment, $V=0.12$

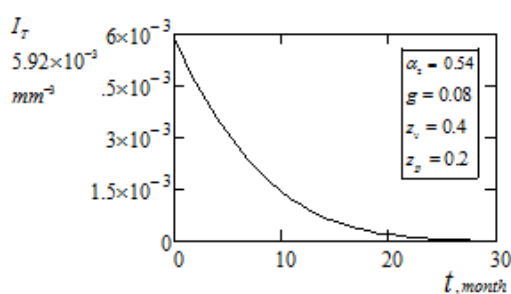

b) Viruses infected CD4 $+\mathrm{T}$ cells with treatment, $I_{T}=0.00592$

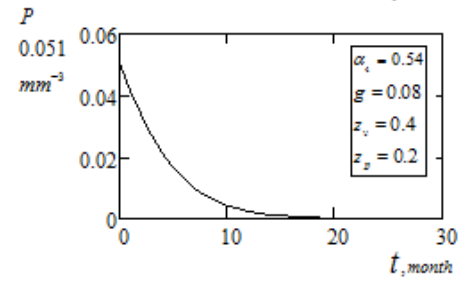

d) Parasitoid-pathogen with treatment, $P=0.051$

Figure 3: Simulation of dual HIV-pathogen infection with treatment initiation, $r(t)=0.5$.

The implication is that, treatment started with strong dose schedule and then diminishes (stable i.e., $Q^{*}=1.122$ ) in strength as infections (viruses) de-replicates after 27 months of treatment.

Furthermore, using equation (2.16), derived from the combination of equations (2.4)-(2.8), coupled with the adjoint system (2.11)-(2.14) and the substituted equation (2.15) for $r^{*}$, we investigate the dynamic optimal control to justify the imposition of the penalty condition on the constraints.

Applying the same variables and parameter values as in figures $3 \mathrm{a}-$ $3 \mathrm{~d}$ and with inclusion of the transversality conditions $\left(\lambda_{1}=0.7, \lambda_{2}=0.2\right.$ $\lambda_{3}=0.1, \lambda_{4}=0.1$ ), into Table 1 , we simulate as in figures $5 \mathrm{a}-5 \mathrm{~d}$ above, without figures of the conditions for brevity.
From Figure 5a above, with lessened amount optimal weight factor of $\psi=0.2$, balanced by control function of $r(t)=0.8$, and imposed transversality conditions, we observed sustainability of maximized healthy $\mathrm{CD}^{+} \mathrm{T}$ cells population of $U_{T}(27)=0.803$, which then suggest near eradication of infected $\mathrm{CD} 4^{+} \mathrm{T}$ cells. The diminishment of infected $\mathrm{CD} 4^{+} \mathrm{T}$ cells i.e., $I_{T}(27)=0$, as shown by figure $5 \mathrm{~b}$, ascertains the claim in figure $5 \mathrm{a}$. As a result of the imposed penalty and the regularization of chemotherapy by the control function, we saw a rapid clearance rate of both viral load and parasitoid-pathogen at the 19 and 18 months (i.e., $V(19)=0$ and $P(18)=0$ ), respectively (Figures $5 c$ and $5 \mathrm{~d}$ ).

From Figure 6 above, we further verify the objective functional of the corresponding chemotherapy administered within the duration 


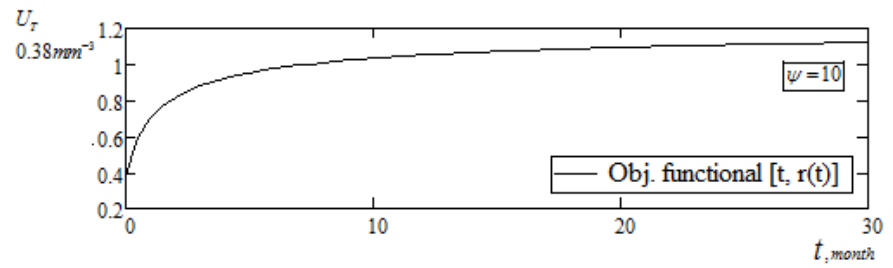

Figure 4: Simulation of control function with treatment initiation, $r(t)=0.5$.

$U_{\tau}$

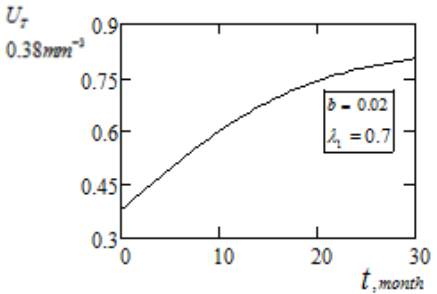

a) Dynamic optimal for uninfected $C D 4+T$ cells, $U_{T}=0.38$

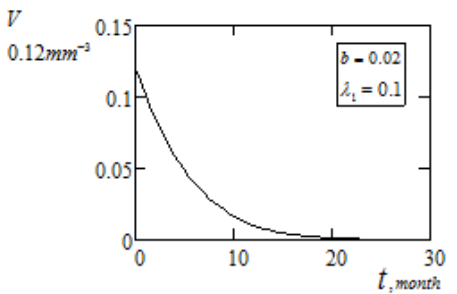

c) Dynamic optimal viral load, $V=0.12$

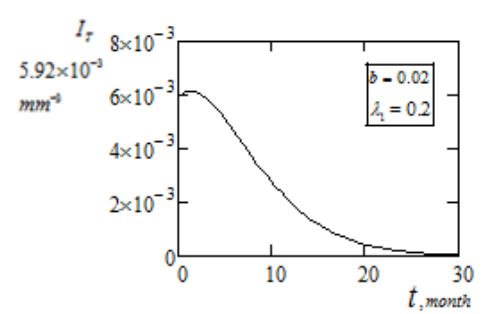

b) Dynamic optimal viruses infected CD4 $+\mathrm{T}$ cells, $I_{\tau}=0.00592$

$P$

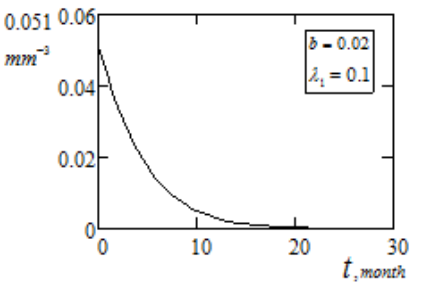

d) Dynamic optimal parasitoid-pathogen, $P=0.051$

Figure 5: Simulation of dynamic optimal control with transversality condition, $r(t)=0.8$.

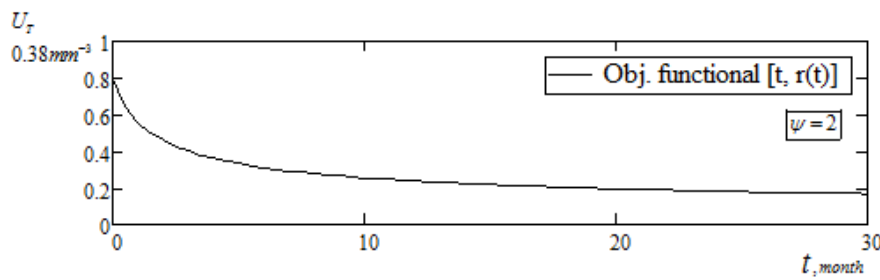

Figure 6: Simulation of dynamic optimal control function, $r(t)=0.8$.

simulated (Figures 5a-5d). With the same initial condition for $U_{T}$, and varying values of $\psi$ and $r(t)$, we validate the trend of the optimality system. Result indicates minimization of benefit cost as drug intensity decline sharply with $Q^{*}=0.167$, representing the remains of infected population who were still subjected to lessened chemotherapy after 27 months of treatment. A summary of the optimal control simulation is as presented in (Table 2).

Where, months - period covered for treatment from set-point of infection, $\psi$ - optimal weight ratio of systemic cost of treatment, $r(t)$ optimal control function, $U_{T(0)}, I_{T(0)}, V_{0}, P_{0}$ - initial condition for treatment initiation, $U_{T}(27)$ - final condition for each treatment duration and $Q$ - objective functional values, $Q=Q\left(r^{*}\right)$.

From Table 2, that there is not much significant difference in final outcome of the optimal benefit of the chemotherapy at the duration of 27 months of treatment, whereas, systemic cost $Q\left(r^{*}\right)$ varies greatly. The implication is that the benefit to the cost control is independent of the intensity of chemotherapy over a prolong time duration (Figures 3 and 5).

\section{Discussion}

We have proposed and formulated a set of mathematical model aimed at controlling the percentage effect of chemotherapy on the $\mathrm{CD} 4^{+}$ T cells following dual viruses' infectivity. The approach is an extension of single HIV infection from a number of HIV literatures as carefully cited in the introductory part of the work. The model is admissible and varies chemotherapy control function, which allowed the study and suppression of viruses infectivity $h_{1}$ and $h_{2}$ respectively. We presented the model using optimal control strategy from which the dynamical optimal control was analytically established. The objective functional that maximized the control system was linearized by the introduction of a simple non-linear control on the cost indicator and penalty term imposed on the constraints, with which the adjoint variables together with transversality conditions were established. The optimality control system is a two-point boundary value problem due to state initial 
Citation: Bassey EB, Kimbir RA and Lebedev KA (2016) On Optimal Control Model for the Treatment of Dual HIV-Parasitoid Pathogen Infection. J Bioengineer \& Biomedical Sci 7: 212. doi: 10.4172/2155-9538.1000212

Page 7 of 7

\begin{tabular}{|l|c|c|c|c|c|c|c|c|}
\hline Experiment & Month & $\boldsymbol{\Psi}$ & $\mathbf{r}(\mathbf{t})$ & $\mathbf{U}_{\mathbf{T}(\mathbf{0})}$ & $\mathbf{U}_{\mathbf{T}(27)}$ & $\mathbf{I}_{\mathbf{T}(\mathbf{0})}$ & $\mathbf{V}_{\mathbf{O}}$ & $\mathbf{P}_{\mathbf{0}}$ \\
\hline Figure 3 & 27 & 10 & 0.5 & 0.38 & 0.802 & 0.00592 & 0.12 \\
\hline Figure 5 & 27 & 2 & 0.8 & 0.38 & 0.803 & 0.0 & 0.0 \\
\hline
\end{tabular}

Table 2: Results of objective functional for model optimality system.

data and adjoint system final time data. Using numerical methods, outcome of analytical determination were numerically validated with compactible experimental data.

Results from simulations affirmed the fact that maximization of immune system and optimal cost on chemotherapy is a function of optimal dynamical control, achievable by regularization of treatment schedule in a fashion dignified by initial high intensity of chemotherapy, measurable by the optimal weight factor and control by the control function over a finite period of time interval.

We further deduced from results that the benefit and effect of chemotherapy were sharper and more effective if initiated at the start of infection set-point. Therefore, it becomes obvious that the effect of chemotherapy diminishes over time duration and as de-replication and mutation of viruses gradually manifest into the system. The consequences are the near stable outcomes of benefits on optimal chemotherapy over prolong treatment duration. Moreso, it is observed that at onset of infection, initialization of treatment with high intensity chemotherapy (i.e. $\psi=10, r(t)=0.5$ ), caused rapid decline of HIV-virus and parasitoid-pathogen and lead to greater recovery of healthy $\mathrm{CD} 4^{+}$ T cells (i.e., $\left.U_{T}(27)=0.38 \rightarrow 0.802\right)$.

However, the greatest optimal control on chemotherapy is experienced on prolong drug dosage application when $U_{T}$, approaches stability following de-replication of viruses. Low value of $\psi$, implies reduction in systemic cost and optimal $r^{*}$ is visible from the objective functional when $Q^{*}$ is maximal.

\section{Conclusion}

In this paper, a 4-Dimensional mathematical model, using ordinary differential equations were formulated. The model accounted for optimal control benefits and methodological treatment of dual HIVparasitoid pathogen infectivity on the host $-\mathrm{CD} 4^{+} \mathrm{T}$ cells count, with RTI, as treatment factor. The method used was analytical optimal control strategy and simulated using numerical methods. From the outcome of model analyses, it is observed that control of viruses' infectivity is a direct function of chemotherapy regularization, achievable by the introduction of optimal weight factor on the objective functional and the imposition of penalty term on the constraints for the attainment of sustainability of maximized healthy $\mathrm{CD} 4^{+} \mathrm{T}$ cells. Furthermore, benefit on cost function is highest with high intensity drug dosage at initiation of onset treatment. On the other hand, results shows that minimization of optimal control on chemotherapy is greatest after a prolong drug schedule over a finite time duration. Non-the-less, for a prolong treatment duration, there exist insignificant variations in the benefits from the control function and thus, maximization of healthy immune system is independent of treatment duration. The model therefore recommends further windows of improvement with the incorporation of multiple immunotherapies which can possibly avert early drug resistance by multiple viruses' infectivity.

\section{References}

1. Fister KR, Lenhart S, Joseph SM (1998) Optimizing Chemotherapy in an HIV Model. Journal of Differential Equations 32: 1-12.

2. Kirschner D (1996) Using Mathematics to Understand HIV Immune Dynamics. Notices of the AMS 43: 191-202.
3. Kirschner D (1999) Dynamics of Co-infection with M. tuberculosis and HIV-1. Theoretical Population Biology 55: 94-109.

4. Bassey BE, Lebedev KA (2015) On Mathematical Modelling of the Effect of $\mathrm{Bi}$-Therapeutic Treatment of Tuberculosis Epidemic. Journal of Mathematics and Statistics 9: 1-7.

5. Zarei H, Kamyad AV, Effati S (2010) Maximizing of Asymptomatic Stage of Fast Progressive HIV Infected Patient Using Embedding Method. Intelligent Control and Automation 1: 48-58.

6. Butler S, Kirschner D, Lenhart S (1995) Optimal Control of the Chemotherapy Affecting the Infectivity of HIV. Mathematical Biology and Medicine, World Scientic 6: 557-569.

7. Perelson AS, Kirschner DE (1993) A Model of the Immune System Response to HIV: AZT Treatment Studies. SFI Working Paper: 1993-04-022.

8. Shirazian M, Farahi MH (2010) Optimal Control Strategy for a Fully Determined HIV Model. Intelligent Control and Automation 1: 15-19.

9. Najariyan M, Farahi MH, Alavian M (2011) Optimal Control of HIV Infection by using Fuzzy Dynamical Systems. J Math Computer Sci 2: 639-649.

10. Wein LM, Zenios SA, Nowak MA (1997) Dynamic Multidrug Therapies for HIV: A Control Theoretic Approach. J Theor Bio 185: 15-29.

11. Bassey EB, Lebedev KA (2016) On Analysis of Parameter Estimation Mode for the Treatment of Pathogen-Induced HIV Infectivity. Open Access Library Journal 3: 1-13.

12. Kirschner D, Webb GF (1996) A Model for Treatment Strategy in the Chemotherapy of AIDS. Bull Math Biol 58: 367-390.

13. Joshi HR (2002) Optimal Control of an HIV Immunology Model. Optimal Control Applications and Methods 23: 199-213.

14. Kirschner D, Webb GF (1998) Immunotherapy of HIV-1 Infection. J Biol Syst 6: 71-83.

15. Ho DD, Neumann AU, Perelson AS, Wen C, John ML, et al. (1995) Rapid Turnover of Plasma Virions and CD4 Lymphocytes in HIV-1 Infection. Nature 273: 123-126.

16. Butler S, Kirschner D, Lenhart S (1997) Optimal Control of Chemotherapy Affecting the Infectivity of HIV.

17. Nara PL (1990) Emergence of Viruses Resistant to Neutralization by V3Specific Antibodies in Experimental HIV Type 1 IIIB Infection of Chimpanzees. J Virol 63: 3779-37791.

18. Kamien MI, Schwarz NL (1991) Dynamic Optimization. 